\title{
Two Important Issues in Subacute Thyroiditis Management: Delayed Diagnosis and Inappropriate Use of Antibiotics
}

\author{
Hayri Bostan Muhammed Erkam Sencar Murat Calapkulu Sema Hepsen \\ Hakan Duger Ilknur Ozturk Unsal Mustafa Ozbek Erman Cakal \\ Department of Endocrinology and Metabolism, Diskapi Yildirim Beyazit Training and Research Hospital, \\ University of Health Sciences, Ankara, Turkey
}

\section{Keywords}

Antibiotic overuse $\cdot$ Late diagnosis · Neck pain · Subacute thyroiditis

\begin{abstract}
Background: Subacute thyroiditis (SAT) is a rare inflammatory disease of the thyroid gland. It has been noticed that patients with a diagnosis of SAT visit more other clinics and receive antibiotics unnecessarily. Therefore, the aim of this study was to reveal the degree of delay in the diagnosis of SAT, prediagnosis antibiotic use rates, and the awareness of clinics for the diagnosis of SAT. Methods: A total of 121 patients with SAT were enrolled in the study. A retrospective analysis was made of the history of patient symptoms during the diagnosis, which physicians they visited, antibiotic use, laboratory test results, and ultrasonographic findings. $\boldsymbol{R e}$ sults: The median age of the patients was 41 years. Neck pain radiating to the jaw/ear was seen in most patients $(71.1 \%)$. The median time from symptom onset to a diagnosis of SAT was 23 days (range, 6-70 days). Antibiotics were erroneously prescribed to 71 patients (58.7\%) before the diagnosis. The median time to diagnosis was 28 days in patients using antibiotics and 20 days in the group not using antibiotics ( $p<$
\end{abstract}

karger@karger.com www.karger.com/etj

2021 European Thyroid Association Published by S. Karger AG, Basel
0.001). Two or more physicians had been visited before SAT diagnosis by 89 (73.6\%) patients, and more antibiotics were prescribed to these patients than the group who visited fewer physicians $(p<0.05)$. The frequency of prescribing antibiotics by physicians was $73.7 \%$ by emergency physicians, $53.1 \%$ by family doctors, $51.1 \%$ by ENT specialists, and $35.4 \%$ by internal medicine specialists. Conclusion: The diagnosis of SAT is often delayed, and misdiagnosis leads to erroneous antibiotic overuse. Physicians should increase their awareness of the diagnosis of SAT in patients with neck pain.

(c) 2021 European Thyroid Association Published by S. Karger AG, Basel

\section{Introduction}

Subacute thyroiditis (SAT) is a self-limiting, inflammatory process of the thyroid gland that often presents with painful swelling of the thyroid. SAT affects females more often than males (female to male ratio of 4:1) [1-3]. The peak age of incidence is $40-50$ years [1]. Although viral infections are thought to trigger the disease, the exact etiology has not been elucidated [4]. Some studies have suggested that certain types of human leukocyte antigens may play a role in the development of SAT $[5,6]$. 
Common initial clinical presentation includes anterior neck pain that can spread up to the jaw or ear and/or down to the sternum, mild to moderate fever, fatigue, malaise, and mild to moderate thyrotoxic symptoms [14]. The affected side of the thyroid gland (unilateral or bilateral) becomes tender to palpation. Atypical cases of SAT without neck pain or tenderness have occasionally been reported in the literature $[2,7,8]$. Some authors have claimed that there is a seasonal peak in SAT cases during the spring and/or early autumn $[1,3]$. Laboratory findings usually include high C-reactive protein (CRP) and erythrocyte sedimentation rate (ESR), suppressed thyroid-stimulating hormone (TSH), and elevated free thyroxin (fT4) levels. Characteristic ultrasound features of SAT include ill-defined heterogeneous, hypoechoic areas and a lack of flow color Doppler in those areas, and in some patients, diffuse enlargement in the thyroid gland may be seen $[9,10]$. These symptoms and laboratory findings are characteristic for the initial (thyrotoxic) phase of SAT.

Despite recent advances in diagnostic methods, the diagnosis of SAT is still often delayed, and patients are unable to continue normal daily activities for weeks or even months. It has been realized that primary care physicians and some specialists do not consider the possibility of SAT in the differential diagnosis of neck pain. Therefore, physicians frequently prescribe antibiotics unnecessarily as the symptoms and laboratory results of the patients can be easily confused with different infective processes. The purpose of this study was to demonstrate the time lag between symptom onset and diagnosis of SAT and to determine the number of doctors who were visited and the frequency of antibiotic use during the diagnosis process. It was also aimed to characterize the frequency of symptoms and laboratory findings in SAT patients.

\section{Materials and Methods}

\section{Study Population and Design}

This retrospective study was designed in the Diskapi Yildirim Beyazit Training and Research Hospital Endocrinology and Metabolism Department. A scan of the database identified 138 patients who were diagnosed with SAT between November 2017 and July 2020. A total of 17 of these patients were excluded due to deficiencies in the laboratory and/or ultrasound examinations, insufficient clinical history before the diagnosis, or no history of neck pain. Thus, a total of 121 patients were enrolled in the study. The data of the patients, including how many and which physician(s) they visited before the diagnosis, antibiotic use, symptoms at the time of diagnosis, the severity of neck pain according to pain scales, laboratory results, ultrasonographic fea- tures at the time of diagnosis, and the treatment they received, were obtained retrospectively from the system records.

The diagnosis of SAT was made based on clinical presentation, physical examination, laboratory test results, and ultrasonographic findings following the recommendations of 2016 American Thyroid Association guidelines [11]. The diagnosis was established with the presence of painful, tender, and hard goiter, elevation of ESR or CRP, elevation of fT4 and suppression of serum $\mathrm{TSH}$, and the presence of hypoechoic areas with blurred margins and decreased vascularization appearance on ultrasonography of painful thyroid regions. Cytological verification was performed in patients with a suspected diagnosis to rule out possible malignancy/painful hashitoxicosis. Besides, improvements in ultrasonographic findings in the posttreatment period were confirmed in all patients. Neck pain was accepted as the initial symptom of the patients to determine the time from symptom onset to diagnosis of SAT.

\section{Laboratory Evaluation}

The laboratory examinations were performed in our institute using a biochemical analyzer. Serum levels of TSH, fT4, free triiodothyronine (fT3), ESR, CRP, and WBC were examined at the time of diagnosis. Thyroid function tests were measured with direct chemiluminescent immunoassay (Beckman Coulter, CA, USA). Reference ranges were defined as TSH: $0.38-5.33 \mathrm{mIU} / \mathrm{L}$, fT4: 0.60-1.25 ng/dL, fT3: 2.28-4 ng/L, ESR: 0-20 mL/h, CRP: 0-5 $\mathrm{mg} / \mathrm{L}$, and WBC: $3,570-11,01010^{3} / \mu \mathrm{L}$. US examinations were obtained using a Hitachi HI Vision Preirus unit (EUB 7000 HV; Hitachi, Tokyo, Japan) with a $13-\mathrm{MHz}$ linear array transducer.

\section{Statistical Analysis}

Statistical analysis was made using SPSS software (version 23.0, SPSS; IBM Corporation, New York, NY, USA). Categorical variables were summarized with frequencies and percentages (\%). Data distribution was assessed with the Kolmogorov-Smirnov test. Data with abnormal distribution were expressed as median (range) and those with normal distribution as mean \pm standard deviation values. Categorical variables were compared using the $\chi^{2}$ test. Continuous variables with normal distribution were compared using the independent samples $t$ test and those not showing normal distribution with the Mann-Whitney $U$ test. The statistical significance level was determined as $p<0.05$.

\section{Results}

A total of 121 patients diagnosed with SAT were included in the final analyses. The median age was 41 years (range, 26-72 years). The female-male ratio was approximately 3:1. The mean pain intensity score, which measures the severity of neck pain in the last $24 \mathrm{~h}$, was high (7.74 \pm 1.56$)$. Neck pain radiating to the jaw/ear was seen in most patients (71.1\%). The vast majority (93.4\%) of patients complained of fatigue. Fever, which was documented at $\geq 38^{\circ} \mathrm{C}$, and palpitations were observed in approximately half of the patients ( 46.3 and $50.4 \%$, respectively), while weight loss, defined as the loss of $\geq 5 \%$ of 
Table 1. Baseline characteristics of the patients $(n=121)$

\begin{tabular}{|c|c|}
\hline Variables & Patients \\
\hline$N(\%)$ & $121(100)$ \\
\hline Age, median (range), years & $41(26-72)$ \\
\hline Female gender, $n(\%)$ & $91(75.2)$ \\
\hline \multicolumn{2}{|l|}{ Hypoecogenic areas at US, $n(\%)$} \\
\hline Bilateral & $62(51.2)$ \\
\hline Unilateral & $59(48.8)$ \\
\hline \multicolumn{2}{|l|}{ Symptoms } \\
\hline Severity of pain, mean \pm SD & $7.74 \pm 1.56$ \\
\hline Spread of pain to jaw/ear, $n(\%)$ & $86(71.1)$ \\
\hline Fatigue, $n(\%)$ & $113(93.4)$ \\
\hline Fever, ${ }^{*} n(\%)$ & $56(46.3)$ \\
\hline Palpitation, $n(\%)$ & $61(50.4)$ \\
\hline Weight loss, $n(\%)$ & $21(17.4)$ \\
\hline Time to diagnosis, median (range), days & $23(6-70)$ \\
\hline Diagnosed at $\geq 15$ days, $n(\%)$ & $99(81.8)$ \\
\hline Presence of antibiotic use, $n(\%)$ & $71(58.7)$ \\
\hline Beta-lactam & $48(67.6)$ \\
\hline Quinolone & $14(19.7)$ \\
\hline Macrolide & $9(12.7)$ \\
\hline Visiting 2 or more physicians, $n(\%)$ & $89(73.6)$ \\
\hline \multicolumn{2}{|l|}{ Thyroid hormone status, $n(\%)$} \\
\hline Hyperthyroid & $104(86.0)$ \\
\hline Euthyroid & $17(14.0)$ \\
\hline TSH, median (range), mIU/L & $0.01(0.001-3.60)$ \\
\hline fT4, median (range), ng/dL & $2.17(0.86-7.77)$ \\
\hline fT3, median (range), ng/L & $5.69(2.83-14.9)$ \\
\hline WBC, median (range), $10^{3} / \mathrm{mm}^{3}$ & $8.9(4.8-16.8)$ \\
\hline $\mathrm{ESR}$, mean $\pm \mathrm{SD}, \mathrm{mm} / \mathrm{h}$ & $57.9 \pm 22.2$ \\
\hline $\mathrm{CRP}$, median (range), mg/L & $69.4(10.1-208.0)$ \\
\hline
\end{tabular}

CRP, C-reactive protein; ESR, erythrocyte sedimentation rate; fT3, free tri-iodothyronine; fT4, free thyroxin; TSH, thyroid-stimulating hormone; US, thyroid ultrasonography; WBC, white blood cells. ${ }^{*} \geq 38^{\circ} \mathrm{C}$ documented fever. ${ }^{9}$ Loss of $5 \%$ or more of body weight in the last month.

body weight in the last month, was present in $17.4 \%$. The median time from symptom onset to diagnosis of SAT was 23 days (range, 6-70 days). There were 22 patients who were diagnosed within 2 weeks of symptom onset (18.2\%). Most of the patients (86.0\%) were in the hyperthyroid phase at the time of diagnosis. The mean ESR value was $57.9 \pm 22.2 \mathrm{~mm} / \mathrm{h}$, and the median CRP value was $69.4 \mathrm{mg} / \mathrm{L}$. The baseline characteristics of the patients are summarized in Table 1.

Antibiotics were erroneously prescribed to 71 patients (58.7\%) by physicians before the diagnosis of SAT. Two or more antibiotics were prescribed to 25 of these 71 patients $(35.2 \%)$ at different times. Beta-lactam group antibiotics had been used by $67.6 \%$ of these patients. The most prescribed antibiotic was amoxicillin plus clavulo- nic acid. The patients were separated into 2 groups as those who received antibiotics and those who did not. The groups were comparable in terms of age, gender, and pain severity $(p>0.05)$. The TSH, fT4, fT3, and ESR levels of both groups were similar $(p>0.05)$. While high CRP levels and frequency of fever and weight loss tended to be seen more in the antibiotic group, these factors did not reach statistical significance $(p=0.16, p=0.12$, and $p=$ $0.07)$. Patients receiving antibiotics visited more doctors than the no-antibiotic group $(p<0.001)$. The median time of diagnosis in the antibiotic group was 28 days, and it was 20 days in the no-antibiotic group, with a statistically significant difference determined between the diagnosis times of the groups $(p<0.001)$. The comparisons of these 2 groups are summarized in Table 2.

Before the diagnosis of SAT, 89 patients had visited 2 or more different physicians (73.6\%). This group that visited more physicians $(\geq 2)$ (MVG) had higher mean neck pain scores $(p=0.014)$ and more fever and palpitation symptoms compared to the group that visited fewer physicians (FVG) $(p<0.05)$. The frequency of antibiotic prescription in the MVG group was $73.0 \%$, which was significantly higher than that of the other group $(p<0.001)$. Five patients had not been treated by another physician before the diagnosis. Antibiotics were prescribed to 6 (22.2\%) of 27 patients examined by only one physician before diagnosis, to $43(65.1 \%)$ of 66 patients examined by 2 physicians, and to $22(95.6 \%)$ of 23 patients examined by 3 or more physicians (Fig. 1). Statistically significant differences were determined between the 2 groups in terms of the levels of TSH with the median value in MVG of $0.01(0.001-3.6)$ and in FVG of $0.03(0.003-3.02)$ $\mathrm{mIU} / \mathrm{L}(p=0.006)$, median fT4 in MVG of $2.32(0.86-$ $7.77)$ and in FVG of $2.00(0.92-5.37) \mathrm{ng} / \mathrm{dL}(p=0.05)$, mean ESR value in MVG of $60.2 \pm 22.5$ and in FVG of 51.3 $( \pm 20.4) \mathrm{mm} / \mathrm{h}(p=0.05)$, and median CRP value in MVG of $76.5(10.1-208.0)$ and in FVG of 45.9 (12.1-132.0) $\mathrm{mg} / \mathrm{L}(p=0.015)$ (Table 3$)$.

The data on which physicians based the prescription or not of antibiotics in 100 of the 121 patients were available in the hospital system records. Before the SAT diagnosis, 48 of 100 patients were examined by an internal medicine specialist, 45 by an ear-nose-throat (ENT) specialist, 33 by the family doctor, 17 by an emergency doctor, and 15 by other specialists (e.g., infectious diseases specialist, pulmonologist, and general surgery specialist) at different times. The frequency of prescribing antibiotics by physicians was $73.7 \%$ by emergency physicians, $53.1 \%$ by family doctors, $51.1 \%$ by ENT specialists, and $35.4 \%$ by internal medicine specialists (Fig. 2). When the 
Table 2. Patient characteristics according to antibiotic use

\begin{tabular}{|c|c|c|c|}
\hline Variables & $\begin{array}{l}\text { Antibiotic group } \\
(n=71)\end{array}$ & $\begin{array}{l}\text { No-antibiotic group } \\
(n=50)\end{array}$ & $p$ value \\
\hline$N(\%)$ & $71(58.7)$ & $50(41.3)$ & - \\
\hline Age, median (range), years & $42.0(30-72)$ & $41.0(26-71)$ & 0.55 \\
\hline Female gender, $n(\%)$ & $54(76.0)$ & $37(74.0)$ & 0.79 \\
\hline \multicolumn{4}{|l|}{ Symptoms } \\
\hline Severity of neck pain, mean \pm SD & $7.80 \pm 1.69$ & $7.64 \pm 1.35$ & 0.57 \\
\hline Fatigue, $n(\%)$ & $68(95.7)$ & $45(90.0)$ & 0.20 \\
\hline Fever, $n(\%)$ & $37(52.1)$ & $19(38.0)$ & 0.12 \\
\hline Palpitation, $n(\%)$ & $38(53.5)$ & $23(46.0)$ & 0.41 \\
\hline Weight loss, $n(\%)$ & $16(22.5)$ & $5(10.0)$ & 0.07 \\
\hline Time to diagnosis, median (range), days & $28(6-65)$ & $20(6-70)$ & $0.02 *$ \\
\hline \multicolumn{4}{|l|}{ Physician visits, $n(\%)$} \\
\hline Diagnosed at first time or one visit & $6(8.4)$ & $26(52.0)$ & $<0.001^{*}$ \\
\hline Two or more visits & $65(91.6)$ & $24(48.0)$ & $<0.001^{*}$ \\
\hline TSH, median (range), mIU/L & $0.01(0.001-3.60)$ & $0.01(0.003-2.45)$ & 0.98 \\
\hline fT4, median (range), ng/dL & $2.17(0.9-7.8)$ & $2.24(0.9-7.6)$ & 0.91 \\
\hline fT3, median (range), ng/L & $6.00(2.9-14.9)$ & $5.46(2.8-14.2)$ & 0.92 \\
\hline WBC, median (range), $10^{3} / \mathrm{mm}^{3}$ & $8.7(4.8-16.8)$ & $9.0(5.3-16.8)$ & 0.48 \\
\hline $\mathrm{ESR}$, mean $\pm \mathrm{SD}, \mathrm{mm} / \mathrm{h}$ & $58.0 \pm 22.8$ & $57.6 \pm 21.7$ & 0.92 \\
\hline $\mathrm{CRP}$, median (range), mg/L & $74.1(10.1-208.0)$ & $67.6(12.0-193.9)$ & 0.16 \\
\hline
\end{tabular}

CRP, C-reactive protein; ESR: erythrocyte sedimentation rate; fT3, free tri-iodothyronine; fT4, free thyroxin; TSH, thyroid-stimulating hormone; WBC, white blood cells. * Statistically significant.

Table 3. Patient characteristics according to number of physician visits

\begin{tabular}{|c|c|c|c|}
\hline Variables & $\begin{array}{l}\text { Fewer visit group } \\
(n=32)\end{array}$ & $\begin{array}{l}\text { More }(\geq 2) \\
\text { visit group } \\
(n=89)\end{array}$ & $p$ value \\
\hline$N(\%)$ & $32(26.4)$ & $89(73.6)$ & - \\
\hline Age, median (range), years & $42.5(29-66)$ & $41.0(26-72)$ & 0.82 \\
\hline Female gender, $n(\%)$ & $23(71.9)$ & $68(76.4)$ & 0.61 \\
\hline \multicolumn{4}{|l|}{ Symptoms } \\
\hline Severity of neck pain, mean \pm SD & $7.16 \pm 1.55$ & $7.94 \pm 1.52$ & $0.014^{*}$ \\
\hline Fatigue, $n(\%)$ & $29(90.6)$ & $84(94.4)$ & 0.46 \\
\hline Fever, $n(\%)$ & $7(21.9)$ & $49(55.1)$ & $0.001^{*}$ \\
\hline Palpitation, $n(\%)$ & $9(28.1)$ & $52(58.4)$ & $0.003^{*}$ \\
\hline Weight loss, $n(\%)$ & $2(6.2)$ & $19(21.3)$ & 0.05 \\
\hline Time to diagnosis, median (range), days & $20.0(7-70)$ & $23.5(6-65)$ & 0.15 \\
\hline Presence of antibiotic use, $n(\%)$ & $6(18.7)$ & $65(73.0)$ & $<0.001^{*}$ \\
\hline Overt hyperthyroidism, $n(\%)$ & $25(78.1)$ & $79(88.8)$ & 0.14 \\
\hline TSH, median (range), mIU/L & $0.03(0.003-3.02)$ & $0.01(0.001-3.6)$ & $0.006^{*}$ \\
\hline fT4, median (range), ng/dL & $2.00(0.92-5.37)$ & $2.32(0.86-7.77)$ & 0.052 \\
\hline fT3, median (range), ng/L & $4.48(2.8-10.1)$ & $6.07(3.0-14.9)$ & 0.11 \\
\hline WBC, median (range), $10^{3} / \mathrm{mm}^{3}$ & $8.5(5.6-14.0)$ & $8.9(4.8-16.8)$ & 0.14 \\
\hline $\mathrm{ESR}$, mean $\pm \mathrm{SD}, \mathrm{mm} / \mathrm{h}$ & $51.3 \pm 20.4$ & $60.2 \pm 22.5$ & 0.05 \\
\hline CRP, median (range), mg/L & $45.9(12.1-132.0)$ & $76.5(10.1-208.0)$ & $0.015^{*}$ \\
\hline
\end{tabular}

CRP, C-reactive protein; ESR, erythrocyte sedimentation rate; fT3, free tri-iodothyronine; fT4, free thyroxin; TSH, thyroid-stimulating hormone; WBC, white blood cells. * Statistically significant. 
Fig. 1. The number of patients relative to how many physicians they visited before the diagnosis and the antibiotic prescription rates for each group $(n=121)$. The figure demonstrates that the frequency of prescribing antibiotics increased in correlation with the number of patients' visits.
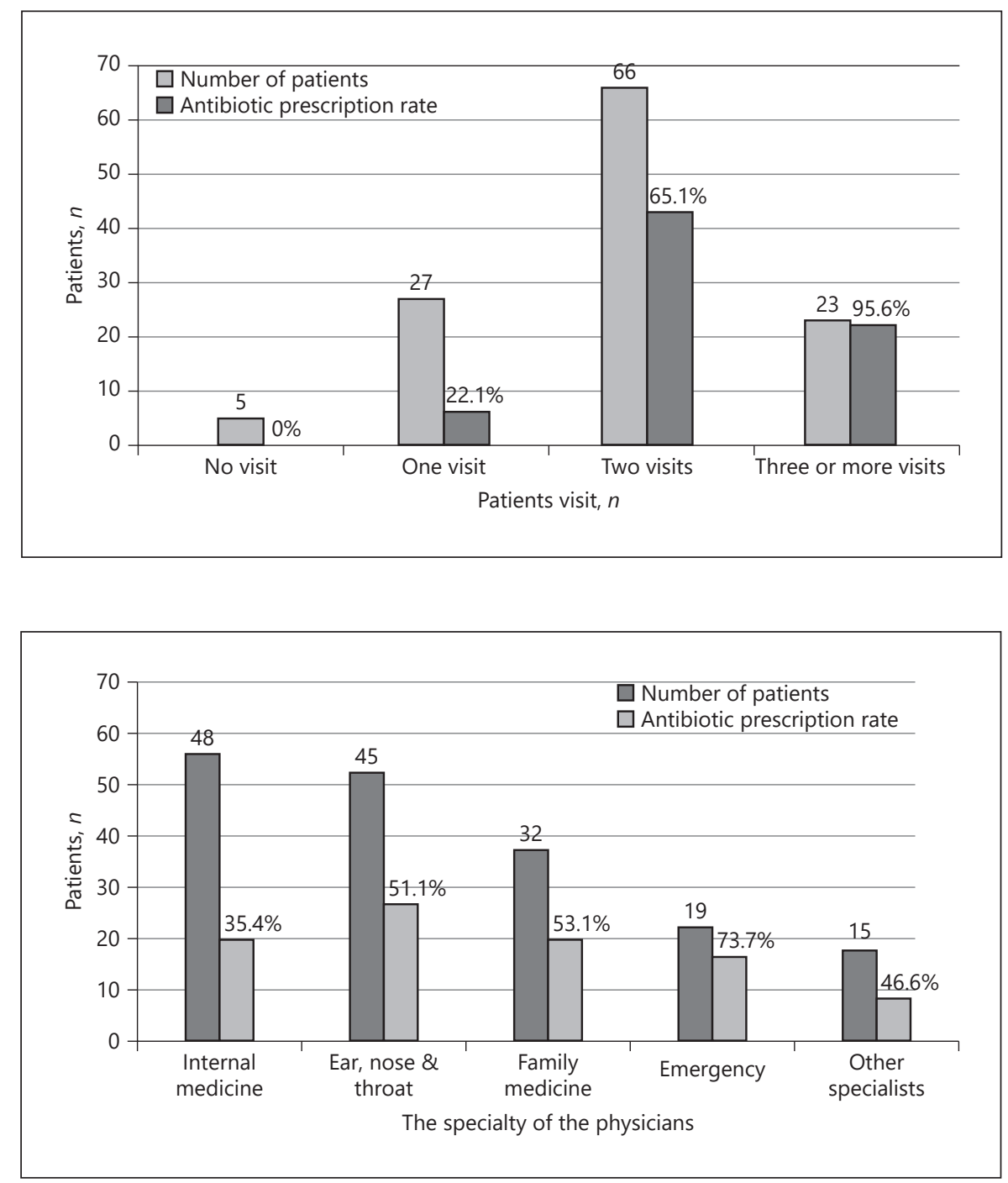

Fig. 2. The number of patients who applied to different specialist groups and the antibiotic prescription rates for each group $(n=100)$. internal medicine physicians and other physicians were compared, ENT, family medicine, and emergency physicians had prescribed more antibiotics significantly (35.4 vs. $56.2 \%, p=0.02$ ).

\section{Discussion}

The diagnosis of SAT is frequently missed as it is a rare disease, and SAT cases tend to occur at the seasonal peak time of upper respiratory tract infections (URI), and similar symptoms may cause confusion [1-3]. In the literature, recent studies have focused on the diagnosis and treatment modalities of SAT. The delays in the prediagnosis period of the disease and its consequences have been presented in only one recent study [12].
The results of this retrospective study showed that the time to diagnosis in $81.8 \%$ of the patients was 2 weeks or more, and the diagnosis of SAT is often delayed. The prediagnosis period was prolonged up to 70 days in some patients. Also, Stasiak et al. [12] reported a delay of $>2$ weeks in SAT diagnosis in $73 \%$ of 64 patients. The pain radiating up to the ear/jaw was observed in $71 \%$ of the patients. Fever and palpitations were present in approximately half of the patients in parallel with findings in the literature, while weight loss was present in a small number of patients [3]. ESR and CRP levels were above the upper limit of normal, similar to data reported in the literature $[1,3,4,13]$. In addition, some authors have suggested that there may be a 1- to 6-week delay between the onset of symptoms and the appearance of laboratory findings $[3,14]$. These results indicate that there are some 
factors contributing to delayed SAT diagnosis. The absence of thyrotoxic symptoms in half of the patients and the normal thyroid function tests in the early stages and the presence of nonspecific symptoms that are confused with other infectious processes seem to be the factors that lead to delays in the diagnosis of SAT. Therefore, in patients presenting with neck pain and who have high acutephase reactants in the laboratory tests, questioning the character and history of the pain (e.g., the presence of pain radiating up to the jaw/ear), performing thyroid function tests, and palpating the thyroid gland in the physical examination are important steps to prevent a delayed diagnosis of SAT.

Delays in the diagnosis of SAT also lead to inappropriate treatment practices. In the current study, $58.7 \%$ of the patients had used antibiotics at least once before the diagnosis of SAT. The most commonly used antibiotics were seen to be from the beta-lactam group, and amoxicillin-clavulonate was the most prescribed antibiotic. It was observed that patients who received antibiotics were diagnosed later and visited clinicians more often than those who did not $(p<0.05)$. The misdiagnosis on the first presentation seems to lead to inappropriate antibiotic use and late diagnosis. Patients whose symptoms did not improve despite receiving antibiotics applied to more physicians, and this, therefore, results in both an increase in healthcare expenditure and a decrease in the functional capacity of the patients.

Stasiak et al. [12] reported that unnecessary antibiotic use was seen in $46.7 \%$ of SAT patients, and patients who received antibiotics had more fever symptoms, more suppressed TSH, and elevated fT4, fT3, WBC, and CRP levels. However, in the current study, there was no significant difference between the groups in terms of symptoms and laboratory findings, which could be attributed to the larger sample size of the current study. But, the main reason for this discrepancy between the 2 studies seems to depend on the factor of the physician. Because of the differences in antibiotic prescription between physicians in our study, heterogeneous distribution of physician's attitudes seems to influence the subgroup analyses. The results of the present study demonstrated that the tendency of the clinician to prescribe erroneously antibiotics in patients with SAT lies in the lack of awareness of the diagnosis of SAT in the differential diagnosis rather than high acute-phase reactants or severity of the clinical features. Also, Stasiak et al. [12] claimed that there was no difference in the diagnosis delay between the groups that received and did not receive antibiotics $(p=0.57)$. However, in the current study, it was shown that unnecessary use of antibiotics causes a delay in the diagnosis of SAT patients as expected.

Unnecessary overuse of antibiotics may cause antibiotic resistance by disrupting the microbiota of the patients and so threaten the individual and public health $[15,16]$. Of the OECD countries with the highest antibiotic resistance, Turkey together with Korea and Greece has a rate of $38.8 \%$ [17]. A recent study demonstrated that antibiotics are most often prescribed in primary care hospitals for uncomplicated respiratory tract infections, and beta-lactam group antibiotics were prescribed most frequently [18]. Antibiotics are not recommended as firstline therapy unless there are complicated respiratory infections (such as bronchitis complicated by pneumonia and culture-proven streptococcal pharyngitis) [19]. Therefore, antibiotics should be given only when necessary, especially in patients presenting with URI symptoms. Inflammatory conditions of the thyroid gland should definitely be included in the differential diagnosis in patients with URI-like symptoms and pain in the anterior neck region.

It was seen in the current study that three-quarters of the patients diagnosed with SAT had consulted at least 2 other physicians before applying to our clinic. Patients in the MVG group had higher neck pain scores and thyrotoxicosis symptoms such as fever, palpitations, and weight loss $(p<0.05)$. ESR and CRP values were significantly higher in the MVG group than in the FVG group. Patients in the MVG group were also more likely to be prescribed antibiotics $(p<0.05)$. While the rate of prescribing antibiotics was $22.1 \%$ in patients who had visited 1 physician before the diagnosis, this rate increased up to $95.6 \%$ in patients who had visited 3 or more physicians. It seems that misdiagnosis on the first presentation resulted in the unnecessary use of antibiotics, and the fact that the symptoms of the patients do not improve leads to repeated visits to different specialists. Moreover, 27.3\% of the patients in the MVG group had used antibiotics 2 or more times. This result indicates that new antibiotics are prescribed for symptomatic patients, and this vicious cycle continues until the correct diagnosis is made.

The specialists most frequently visited by patients before the diagnosis were internal medicine, ENT, family, and emergency physicians, respectively. While emergency physicians stand out as the group that prescribed erroneously antibiotics most frequently $(73.7 \%)$, ENT and family physicians prescribed antibiotics for approximately half of the patients (51.1 and $53.1 \%$, respectively), and internal medicine specialists prescribed antibiotics for approximately $35.4 \%$ of the patients. These results dem- 
onstrate that the diagnosis of SAT is not suspected in most patients on the first presentation. Therefore, internal medicine, ENT, family, and emergency physicians, to whom patients frequently present first, should increase their awareness of this issue. Thus, delays in the diagnosis of SAT and the unnecessary use of antibiotics can be prevented.

There were some limitations to the study. Due to the retrospective nature of the study, there may have been some deficiencies in the patient files, and the results may have been affected by patient-related factors (such as problems remembering symptoms and treatment).

In conclusion, the diagnosis of SAT is often delayed, thereby limiting the functional capacity of patients for a long time, and misdiagnosis leads to inappropriate use of antibiotics. It seems that the factor underlying this inappropriate approach is the low awareness of physicians. Therefore, clinicians who may encounter these patients frequently should increase their awareness of the diagnosis of SAT in patients with neck pain.

\section{Statement of Ethics}

The study was conducted ethically in accordance with the World Medical Association Declaration of Helsinki. Approval for this study was granted by the Ethics Committee of the University of Health Sciences, Diskapi Yildirim Beyazit Training and Research Hospital (Approval No. 95/09). Written consent was obtained from all participants before the study procedures.

\section{Conflict of Interest Statement}

The authors have no conflicts of interest to declare.

\section{Funding Sources}

The authors did not receive any funding for the current study.

\section{Author Contributions}

H.B. and M.E.S. designed the study; all authors contributed to intellectual content; H.B., M.C.., S.H., and H.D. collected data; H.B. and M.E.S. wrote the manuscript; and all authors reviewed the manuscript.

\section{References}

1 Samuels MH. Subacute, silent, and postpartum thyroiditis. Med Clin North Am. 2012 Mar;96(2):223-33.

2 Fatourechi V, Aniszewski JP, Fatourechi GZE, Atkinson EJ, Jacobsen SJ. Clinical features and outcome of subacute thyroiditis in an incidence cohort: Olmsted County, Minnesota, Study. J Clin Endocrinol Metab. 2003 May;88(5):2100-5

3 Nishihara E, Ohye H, Amino N, Takata K, Arishima T, Kudo T, et al. Clinical characteristics of 852 patients with subacute thyroiditis before treatment. Intern Med. 2008;47(8): 725-9.

4 Alfadda AA, Sallam RM, Elawad GE, Aldhukair H, Alyahya MM. Subacute thyroiditis: clinical presentation and long term outcome. Int J Endocrinol. 2014;2014:794943.

5 Nyulassy S, Hnilica P, Buc M, Guman M, Hirschova V, Stefanovic J. Subacute (de Quervain's) thyroiditis: association with HLABw35 antigen and abnormalities of the complement system, immunoglobulins and other serum proteins. J Clin Endocrinol Metab. 1977 Aug;45(2):270-4.

6 Stasiak M, Tymoniuk B, Michalak R, Stasiak B, Kowalski ML, Lewiński A. Subacute thyroiditis is associated with HLA-B* 18 : 01 ,DRB1* 01 and-C* 04: 01: the significance of the new molecular background. J Clin Med. 2020 Feb;9(2):534
7 Daniels GH. Atypical subacute thyroiditis: preliminary observations. Thyroid. $2001 \mathrm{Jul}$; 11(7):691-5.

8 Tsai CH, Lee JJ, Liu CL, Tzen CY, Cheng SP. Atypical subacute thyroiditis. Surgery. 2010 Mar;147(3):461-2.

9 Cappelli C, Pirola I, Gandossi E, Formenti AM, Agosti B, Castellano M. Ultrasound findings of subacute thyroiditis: a single institution retrospective review. Acta Radiol. 2014 May;55(4):429-33.

10 Sencar ME, Calapkulu M, Sakiz D, Akhanli P Hepsen S, Duger H, et al. The contribution of ultrasonographic findings to the prognosis of subacute thyroiditis. Arch Endocrinol Metab. 2020 May-Jun;64(3):306-11.

11 Ross DS, Burch HB, Cooper DS, Greenlee MC, Laurberg P, Maia AL, et al. 2016 American Thyroid Association guidelines for diagnosis and management of hyperthyroidism and other causes of thyrotoxicosis. Thyroid. 2016 Oct;26(10):1343-421.

12 Stasiak M, Michalak R, Stasiak B, Lewiński A. Time-lag between symptom onset and diagnosis of subacute thyroiditis: how to avoid the delay of diagnosis and unnecessary overuse of antibiotics. Horm Metab Res. 2020 Jan;52(1): $32-8$.

13 Sencar ME, Calapkulu M, Sakiz D, Hepsen S, Kus A, Akhanli P, et al. An evaluation of the results of the steroid and non-steroidal antiinflammatory drug treatments in subacute thyroiditis in relation to persistent hypothyroidism and recurrence. Sci Rep. 2019 Nov; 9(1):16899-8.
14 Tachibana T, Orita Y, Ogawara Y, Matsuyama Y, Abe I, Nakada M, et al. Time-lag between symptom onset and laboratory findings in patients with subacute thyroiditis. Auris Nasus Larynx. 2014 Aug;41(4):369-72.

15 Langdon A, Crook N, Dantas G. The effects of antibiotics on the microbiome throughout development and alternative approaches for therapeutic modulation. Genome Med. 2016 Apr;8(1):39.

16 Khanna S, Tosh PK. A clinician's primer on the role of the microbiome in human health and disease. Mayo Clin Proc. 2014 Jan;89(1): 107-14.

17 Isler B, Keske S, Aksoy M, Azap ÖK, Yilmaz $\mathrm{M}$, Yavuz SŞ, et al. Antibiotic overconsumption and resistance in Turkey. Clin Microbiol Infect. 2019 Jun;25(6):651-3.

18 Chang Y, Chusri S, Sangthong R, McNeil E $\mathrm{Hu}$ J, Du W, et al. Clinical pattern of antibiotic overuse and misuse in primary healthcare hospitals in the southwest of China. PLoS One. 2019 Jun;14(6):e0214779.

19 Harris AM, Hicks LA, Qaseem A. Appropriate antibiotic use for acute respiratory tract infection in adults: advice for high-value care from the American College of Physicians and the Centers for Disease Control and Prevention. Ann Intern Med. 2016 Mar;164(6):42534. 